\title{
Central Nervous System Erdheim-Chester Disease
}

National Cancer Institute

\section{Source}

National Cancer Institute. Central Nervous System Erdheim-Chester Disease. NCI

Thesaurus. Code C129808.

Rare involvement of the central nervous system by Erdheim-Chester disease. 